\title{
Organization of Uniform Dispersal for Group of Small Satellites After Their Separation and Acceptable Spread at Stages of Their Further Approaches
}

\author{
Alexander Degtyarev, Irina Vorobiova, Anatoliy Sheptun \\ Yuzhnoye SDO, Dnepropetrovsk, Ukraine \\ Email address: \\ info@yuzhnoye.com (I. Vorobiova)

\section{To cite this article:} \\ Alexander Degtyarev, Irina Vorobiova, Anatoliy Sheptun. Organization of Uniform Dispersal for Group of Small Satellites After Their \\ Separation and Acceptable Spread at Stages of Their Further Approaches. American Journal of Aerospace Engineering. \\ Vol. 2, No. 5, 2015, pp. 36-42. doi: 10.11648/j.ajae.20150205.11
}

\begin{abstract}
Mutual-relative motion of satellites constellation after their placing into initial orbit is characterized the satellites reiterated approaches to minimal distances. Determined separation conditions for each satellite pair in constellation, which realization leads to minimal satellites approaches. It was sustained, that distances between satellites can reach extreme minimal values due to minor angular deviations of orbit planes and always occurring flight altitudes long-period oscillations. Received analytical relations for estimation of angular deviations and distances between satellites orbit planes at the phases of periodical approaches. It was approved that in some vicinity of orbit numbers can occur following almost simultaneously: small differences of angular distances from the equator for any satellite pair in constellation; minimal angular deviations of satellites orbit planes (in general they have different precession after separation of different directions and with various velocities). Analytical relationships were numerically confirmed with using software for satellites movement prediction at the phases of their approaches to the minimum distances. The area of satellites separation parameters was built, with its implementation in a small vicinity of flight orbits the satellites approach to distances less than $100 \mathrm{~m}$. The minimum distance in the specified area according to the single calculations is $\sim 10 \mathrm{~m}$.
\end{abstract}

Keywords: Group Launches, Minimal Distances, Satellites Spatial Separation, Angular Spatial Separation of Orbit Planes, Estimation of Maximal Satellites Approaches, Maximal Satellites Approaches

\section{Introduction}

\subsection{Basic Assumptions}

The tendency of reducing weights and dimension of near space satellites, considerable rocket capabilities and new technologies allow ensuring injection of fair quantities of satellites by one launch. Issues concerning organization of uniform dispersal for constellation of small satellites after their separation and acceptable spread at stages of their further approaches are topical in the rocket building industry. Physical movement foundations in the terrestrial gravitational field determine satellites periodical approaches. Such issues occur two times per orbit in case satellites separation along the normal to orbital velocity and more rarely (up to several thousands of orbits) in case separation along flight direction (fig. 1 shows satellites approaches during their autonomous flight with differences between their separation velocities $0.75 \mathrm{~m} / \mathrm{s}$ ).

In case of small number of satellites in constellation their separation velocities are differ from each other by $1-2 \mathrm{~m} / \mathrm{s}$. The mutual relative motion analysis of satellites constellation and their dispersal during first flight orbits after separation is sufficient. Such analysis method is acceptable for practice. However, relative separation velocities are reducing with growth the number of satellites in constellation (10-20 and more). As a result difficulties of ensuring organization of uniform dispersal for small satellites constellation occur during their first flight orbits after separation. The necessity of satellite approaches estimation during long time intervals occurs at the same time.

The conditions of uniform dispersal for satellite constellation (the number of objects is 12, fig. 2) during their first flight orbits were determined and analytical relations for analysis satellites approaches during long time intervals were preliminary obtained. They were presented in articles $[2,5]$ 
and in the reports $[3,4]$ at $64^{\text {th }}$ (Beijing) and $65^{\text {th }}$ (Toronto) International Astronautical Congresses. Numerical evaluations of satellites approaches for their single separation along the flight direction were also presented. There were noticed satellite approaches on the distances near $100 \mathrm{~m}$. The results of such calculations forces to carrying out of more detailed researches about mentioned issues.

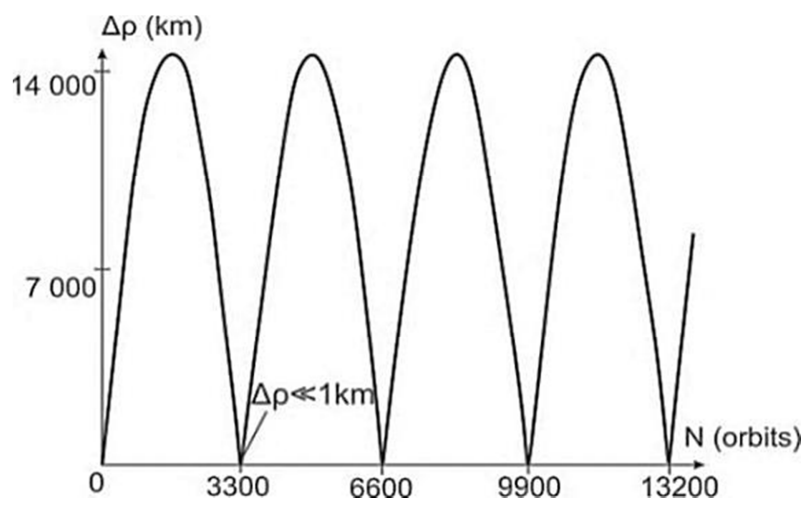

a)

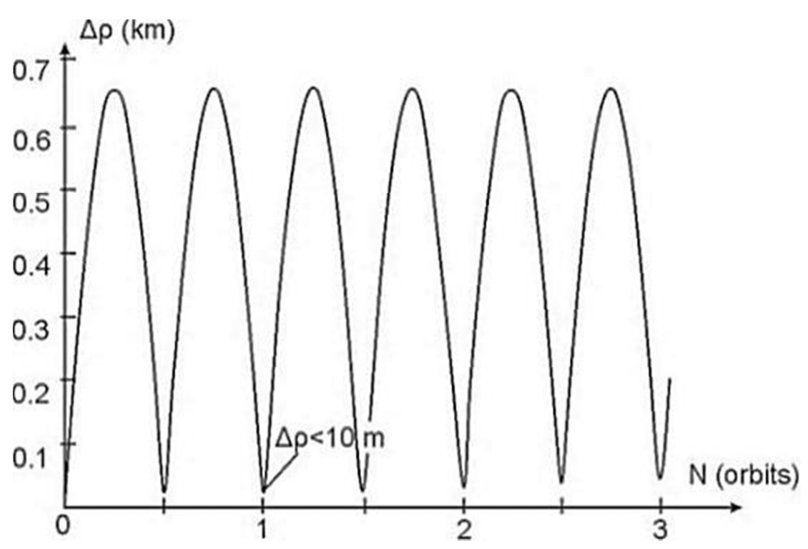

b)

Fig. 1. The typical picture of changing of distances between satellites: a) separation along normal to orbital velocity vector b) separation along flight direction.

The conditions which correspond to satellites approaching on minimal distances for a long time intervals have not researched before.

\subsection{Problem Statement}

The research task is definition of conditions which implementation leads to reducing distances between satellites at the phases of their maximal approaches and obtaining data of numerical evaluation of these distances. Current researches continue ballistic analysis of mutualrelated motion for satellites constellation (12 objects) after their separation from side surface of dispenser normal to longitudinal axis of launch vehicle (fig. 2), which was started in [2-5]. It was presumed that longitudinal axis of dispenser specified along radius-vector. It should be noted that dispencer longitudinal axis $\mathrm{OX}_{1}$ was set along nadir direction.
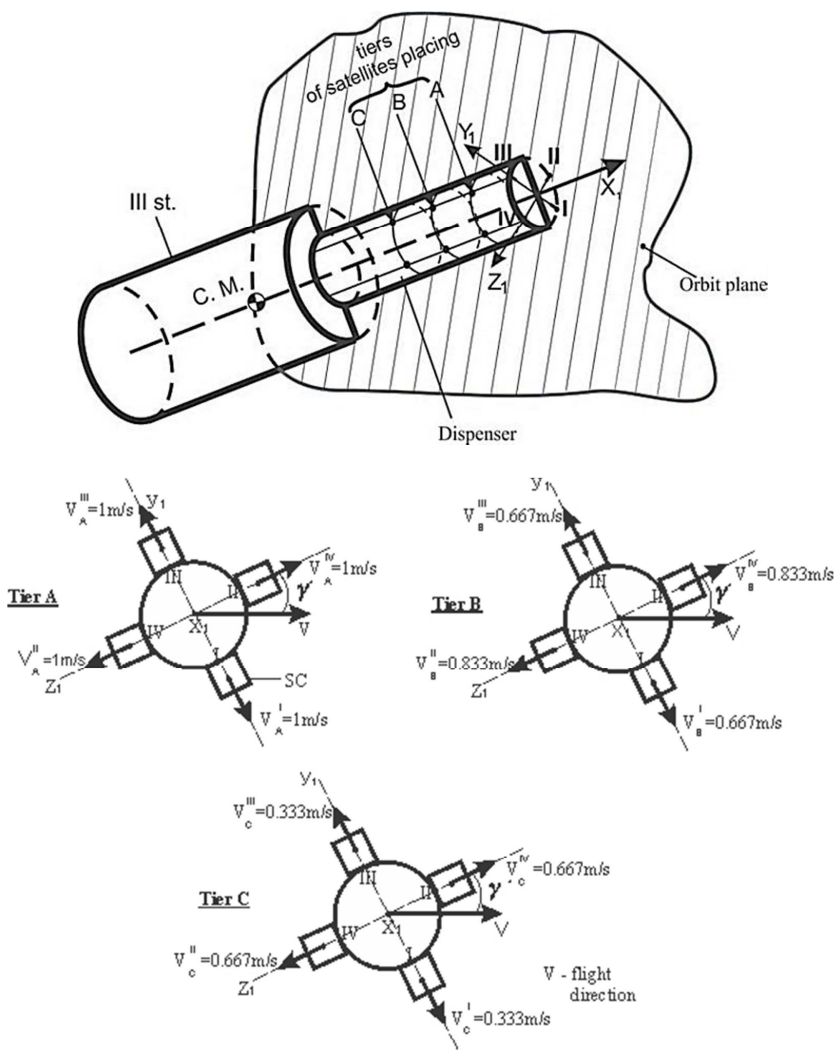

Fig. 2. The scheme of satellites location on dispenser's surface.

The conditions of satellites constellation separation after putting them into initial orbit which ensure uniform dispersal with step of $\sim 2.6 \mathrm{~km}$ along orbit for each flight orbit (fig. 3, table 1) were determinedin [2].

Table 1. Directions and satellites separation velocities $(\mathrm{m} / \mathrm{s})$.

\begin{tabular}{lllll}
\hline \multirow{2}{*}{ Platformindex } & \multicolumn{5}{l}{ Stabilizationplanes } & \multicolumn{5}{l}{ Satellites separation velocities } \\
\cline { 3 - 6 } & I & II & III & IV \\
\hline $\mathrm{A}$ & $\mathrm{V}$ & $\mathrm{V}$ & $\mathrm{V}$ & $\mathrm{V}$ \\
$\mathrm{B}$ & $0.677 \cdot \mathrm{V}$ & $0.833 \cdot \mathrm{V}$ & $0.677 \cdot \mathrm{V}$ & $0.833 \cdot \mathrm{V}$ \\
$\mathrm{C}$ & $0.333 \cdot \mathrm{V}$ & $0.677 \cdot \mathrm{V}$ & $0.333 \cdot \mathrm{V}$ & $0.677 \cdot \mathrm{V}$ \\
\hline
\end{tabular}

The estimation of satellite pairs approaching in constellation were carried out on the base of calculation results of satellites movement after their separation, in case of putting them into sun-synchronous orbits with altitude $\sim 650 \mathrm{~km}$ and inclination $98^{\circ}$ by one launch.
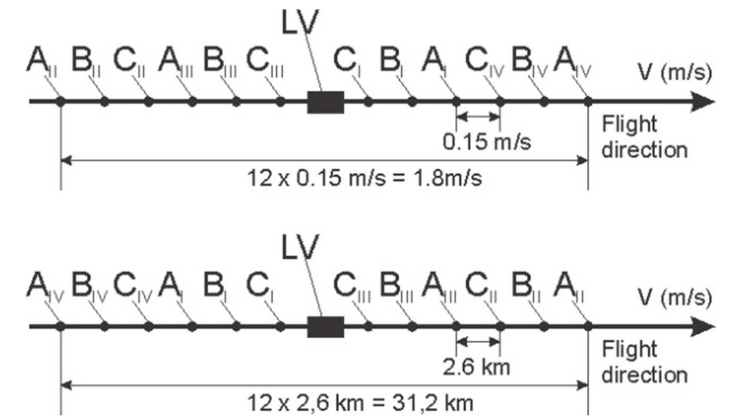

Fig. 3. Satellites separation scheme and their location in flight $\bar{V}=1 \mathrm{~m} /$ s)a) after separationb) by the end of first flight orbit. 
Application Satellite Tool Kit was used for required calculations.

\section{Analytical Relations}

It is examined mutual-relative motion of small satellites constellation with equal ballistic coefficients after putting them into frequently used orbits with low aerodynamic drag $(h>600 \mathrm{~km})$ by one launch. General characteristic of each satellite flight orbit after separation is small differences of semi axis dimensions and inclination (in most cases to $1 \mathrm{~km}$ and $10^{-4} \mathrm{rad}$ correspondently). As a result parameters of satellites orbit planes precession motion differ insignificantly. Satellites approaches on minimal distances during long time intervals are also possible.

Location of two of satellites orbits after their separation and at the expiration some number of flight orbits $N \sim N_{i, j}$ showed at fig. 4,5 .

The distances between satellites decrease and reach their minimal values in some vicinity of flight orbits $N \sim N_{i, j}$ after reaching of the maximum distances between any pair $(i, j)$ of satellites in constellation $\left(\rho_{i, j}=r_{i}+r_{j} \sim 14\right.$ thousands of $\mathrm{km}$, where $r_{i, j}$ are orbital radiuses):

$$
N \sim N_{i, j}=\frac{T_{i}}{\left|T_{i}-T_{j}\right|} \sim \frac{1}{3} \frac{V_{0}}{\Delta V_{V_{i, j}}},
$$

( $T_{i}, T_{j}$ - orbital periods of two $(i, j)$ satellites, $V_{0}-$ flight velocity at the initial orbit, $\Delta V_{V_{i, j}}$ - the difference of separation velocities projections of satellites pair along flight direction).

General number $l$ of satellite pair combinations determines as $l=\frac{n(n-1)}{2}=78$ in case of number of flight objects equal to $n=13$ (constellation of 12 satellites and the dispenser).

The distances between satellites in constellation at phases of minimal distances in the vicinity of orbit number $N \sim N_{i, j}$ are determined by angular spacing $\Delta\left(\delta \psi_{1}\right)_{i, j}^{N_{i, j}}$ of orbital planes $\left(\delta \psi_{1}-\right.$ orbital plane rotation angle per one flight orbit), by changing of average altitudes owing to atmospheric resistance, flight altitudes periodic changes, etc.

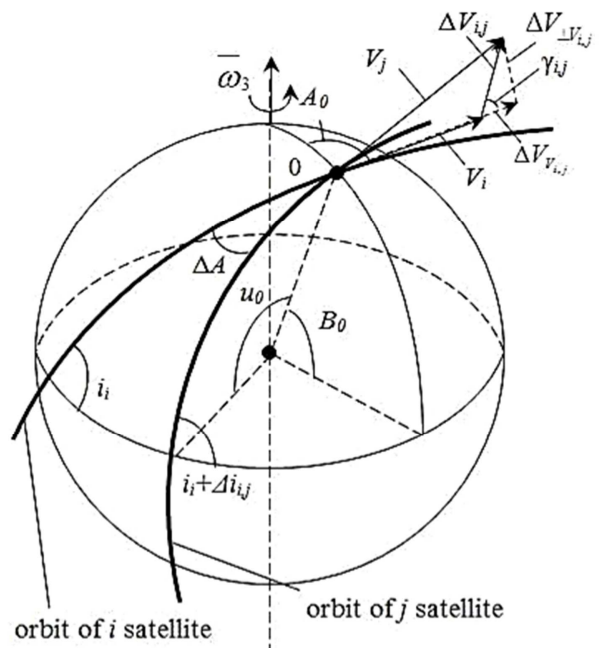

Fig. 4. Location of two satellites orbits after their separation.

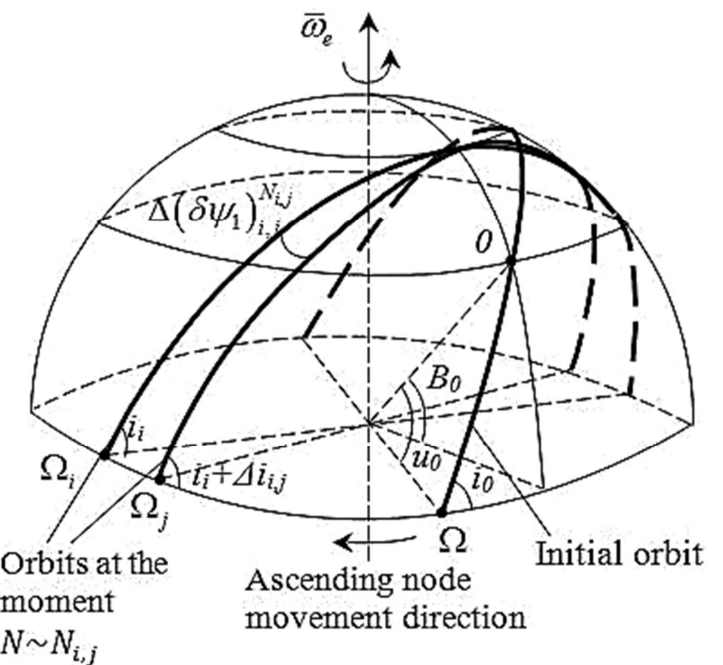

Fig. 5. Location of two satellites orbits at the expiration some number of flight orbits $N \sim N_{i, j}$

Relations for estimation of orbital planes angular spacing $\Delta\left(\delta \psi_{1}\right)_{i, j}^{N_{i, j}}$, linear shifts along lateral direction $\Delta Z_{i, j}^{N_{i, j}}$ and changings of orbital average altitudes $\Delta H_{i, j}^{N_{i, j}}$ for two of satellites in constellation in the vicinity of orbit number $N \sim N_{i, j}$ are presented below:

$$
\begin{gathered}
\Delta\left(\delta \psi_{1}\right)_{i, j}^{N_{i, j}}=\pi\left(\frac{a_{e}}{r_{0}}\right)^{2} \alpha\left[\frac{7}{6} \sin 2 i_{0}-\delta i_{i, j} N_{i, j} \cos 2 i_{0}\right] \\
\Delta Z_{i, j}^{N_{i, j}}=r_{0} \Delta\left(\delta \psi_{1}\right)_{N_{i, j}} \cos u_{0} \\
\Delta H_{i, j}^{N_{i, j}}=\frac{8}{3} \pi B_{k} r_{0}^{3} \frac{\partial \rho\left(h_{0}\right)}{\partial h}
\end{gathered}
$$

(index «0» refers to initial orbit parameters)

Using expressions for $\Delta i_{i, j}, \Delta r_{i, j}, N_{i, j}$ from [1-5] and spherical geometry formulas the first relation in (1) was transformed into:

$\Delta\left(\delta \psi_{1}\right)_{i, j}^{N_{i, j}}=\frac{\pi}{3}\left(\frac{a_{e}}{r_{0}}\right)^{2} \alpha \sin 2 i_{0}\left[\frac{7}{2}-\frac{\Delta V_{\perp V_{i, j}}}{\Delta V_{V_{i, j}}} \operatorname{ctg} 2 i_{0} \cos u_{0}\right]$

( $\Delta V_{\perp V_{i, j}}$ - the difference of separation velocities projections of any satellites pair $(i, j)$ in constellation along the normal to flight direction $V_{0} ; u_{0}$-point on the orbit, which corresponds to angular distance from the equator at the moment of satellites constellation separation (fig. 4)).

First summand in (2) characterizes influence of different average flight altitudes of two satellites onto orbital precession parameters and as a result onto angular dispersal of orbital planes in the vicinity of flight orbit number $N \sim N_{i, j}$. Second summand in (2) characterizes influence of different initial orbit inclinations after satellites separation. Also next statements follow from (2):

- Angular dispersal of any satellite pair $(i, j)$ in constellation are caused by the difference of separation velocities projections $\Delta V_{V_{i, j}}$ in the vicinity of flight orbit number $N \sim N_{i, j}$. Such dispersal are equal to each other 
and in accepted calculation conditions are:

$$
\Delta\left(\delta \psi_{1}\right)_{i, j}^{N_{i, j}}=\frac{7}{6} \pi\left(\frac{a_{e}}{r_{0}}\right)^{2} \alpha \sin 2 i_{0} \sim 9.5 \text { ang. min }
$$

- The usage of satellites separation angularly to initial orbit plane $\left(\Delta i_{i, j} \neq 0\right)$ leads to changing (increase or decrease) angular dispersal of two satellites orbits. Assimilation to zero the expression in brackets in (2) leads to satellites orbit planes correspondence accurate within $\Delta i_{i, j}$ in cafe of realization next condition:

$$
\frac{\Delta V_{\perp V_{i, j}}}{\Delta V_{V_{i, j}}}=\frac{7}{2} \frac{\operatorname{tg} 2 i_{0}}{\cos u_{0}}
$$

Relation obtained below follows from (3) for sunsynchronous orbits $\left(i_{0}=98^{\circ}\right)$ and satellites separation at the equator $u_{0}=0$ :

$$
\frac{\Delta V_{\perp V_{i, j}}}{\Delta V_{V_{i, j}}}=1.0023
$$

Relation (3) affects more onto increasing of two satellites $(i, j)$ approach frequency to minimal distances in the vicinity of flight orbit number $N \sim N_{i, j}$. In case of condition (3) fulfillment the angle between satellites orbit planes does not exceed of angular minutes splits in both directions during valuable orbits number related to 'approaching event' time. This implies that frequency of events of satellites approaching to minimal distances increases.

For example, for sun-synchronous orbits $\left(i \sim 98^{\circ}\right)$ with $600-700 \mathrm{~km}$ altitude in case of condition (3) fulfillment the angular distance between satellites orbit planes does not exceed of 0.2 - 0.3 angular minutes during 200 flight orbits.

There were determined separation conditions (separation velocities and inclinations) for any satellite pair $(i, j)$ in constellation, which realization leads to minimal satellites approaches. In particular it was approved that in some vicinity of orbit numbers can occur almost simultaneously following:

- Small differences of angular distances from the Equator for any satellite pair $(i, j)$ in constellation;

- Minimal angular deviations of satellites orbit planes (in general they have different precession after separation of divers directions with various velocities).

Satellites approaches occur during further flight after their first approaching event. The number of such events $k_{i, j}$ for each satellite pair determines as integer part of relation $k_{i, j}=\left[\frac{N_{\Sigma}}{N_{i, j}}\right]$, where $N_{\Sigma}-$ the number of flight orbits during active satellite exploitation time. This relation for accepted conditions of satellite separation approximately is of the form $k_{i, j}=\left[10,5 \cdot \Delta V_{V_{i, j}}\right]$. The range of $k_{i, j}$ value changing (during 5 year exploitation period, which corresponds to $N_{\Sigma} \sim 27000$ orbits) for every possible satellite pairs in constellation is from 1 to 19 approaching events. General approaches number for all 78 satellite pairs during mentioned time interval is 551 .

Angular orbit planes dispersal of two $(i, j)$ satellites at the moment their $k_{i j}$ approach to minimal distances can be written as $\Delta\left(\delta \psi_{1}\right)_{i, j}^{k N_{i, j}}=k \cdot \Delta\left(\delta \psi_{1}\right)_{i, j}^{N_{i, j}}$ besides condition of angular orbit dispersal minimization [3] will be constant.

It should be noted that orbit inclinations would not change with dispenser longitudinal axis angular placing normal to orbit plane and maintaining of conditions showed at fig. 2 and table 1. As a result conditions (3) for minimal angular deviation are not implemented in some vicinity of flight orbit $N \sim N_{i, j}$. With increase of satellites approaching events number the expression of inaccuracy angular orbit planes dispersal $\Delta\left(\delta \psi_{1}\right)_{i, j}^{k N_{i, j}}$, which can be represented as a linear subjection from number $k_{i j}$ of approaching events, decreases. As a result the accuracy of formula (4) for $k_{i j}$ of approaching event decreases.

\section{Numerical Estimation of Minimal Distances}

The calculations were carried out for sun-synchronous orbits with $\sim 650 \mathrm{~km}$ altitude for constellation composed of 12 satellites $^{2-5}$, which were uniform set on the circle of dispenser in points I, II, III, IV at tiers A, B, C. Satellites separation was accepted at the equator $\left(u_{0}=0\right)$ normal to dispenser longitudinal axis (the angular orientation of it is presented at fig. 2). Velocities and separation directions implement satellite uniform dispersal during first flight orbits and their numerical values were accepted according to data from fig. 2 .

The calculations of flight trajectory for approach evaluation of satellite pair $(i, j)$ in constellation in some vicinity of number $N \sim N_{i, j}=\frac{1}{3} \frac{V_{0}}{\Delta V_{V_{i, j}}}$ were carried out considering change of initial movement conditions due to satellites separation from dispenser. The differences of relative separation velocities $\Delta V_{V_{i, j}}$ and of inclinations $\Delta i_{i, j}$ of satellite pair $(i, j)$ in constellation, which were accepted for calculations according to initial data (table $1, \mathrm{~V}=1 \mathrm{~m} / \mathrm{s}$ ), are showed in table 2. Also there were showed data of full orbit numbers $\left(N_{i, j}\right)$ to the moment of first satellite pair $(i, j)$ approach and count $k_{i, j}$ of approach events.

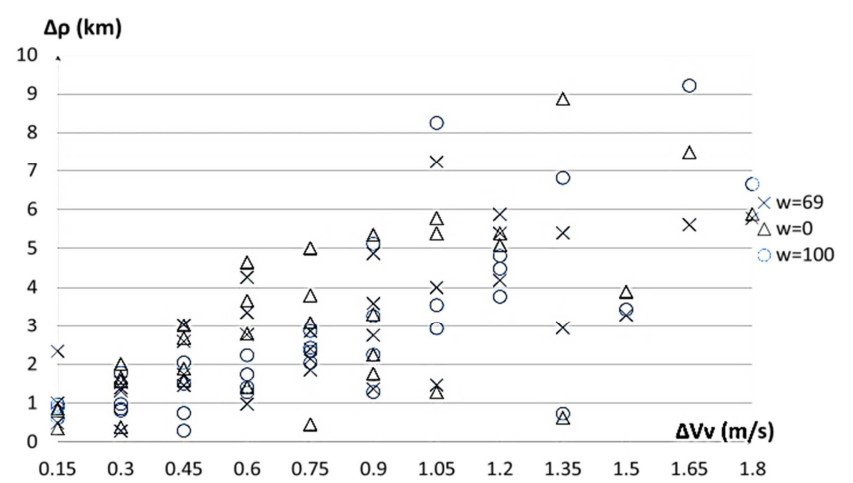

Fig. 6. Distances between satellites at the moment of their first approach in some vicinity of orbit number $N \sim N_{i, j}$ (nominal separation conditions).

The calculations for three values pericentre argument $\omega_{0}=0^{\circ} ; 69^{\circ} ; 100^{\circ}$ were carried out for pericentre argument 
initial value influence of initial orbit with excentricity equal $e_{0}=0.00115\left(\omega_{0}=69^{\circ}\right.$ and $e_{0}=0.00115$ with $h_{0} \sim 650 \mathrm{~km}$ altitude satisfy stable near-Earth orbits, which parameters $\omega_{0}$ and $e_{0}$ remain constant in ascending nodes for long time intervals).

The numbers of distances $\rho_{i, j}$ between satellite pair $(i, j)$ in some vicinity of number $N \sim N_{i, j}$ on the assumption of nominal separation conditions are showed at fig. 6 .

Next conclusion can be drawn from fig. 6, that low border of minimal distance between satellites with nominal separation conditions is at $0,5 \mathrm{~km}$ level in difference range of relative separation velocities $\Delta V_{V_{i, j}}=0,15-1,35 \mathrm{~m} / \mathrm{s}$. Nominal values of satellites minimal distances increase with time.

Table 2.Combination of satellite pair $(i, j)$ separation parameters differences.

\begin{tabular}{|c|c|c|c|c|c|c|c|c|c|c|c|c|}
\hline$\Delta V_{V_{i, j}}(\mathrm{~m} / \mathbf{s})$ & 0.15 & 0.3 & 0.45 & 0.6 & 0.75 & 0.9 & 1.05 & 1.2 & 1.35 & 1.5 & 1.65 & 1.8 \\
\hline$\Delta i_{i, j}(\mathrm{rad})$ & $\begin{array}{l}10^{-5} \\
4 \cdot 10^{-5} \\
1.6 \cdot 10^{-5}\end{array}$ & $\begin{array}{l}2 \cdot 10^{-5} \\
8 \cdot 10^{-5} \\
1.2 \cdot 10^{-4} \\
1.7 \cdot 10^{-4}\end{array}$ & $\begin{array}{l}8 \cdot 10^{-5} \\
1.2 \cdot 10^{-4} \\
1.8 \cdot 10^{-4}\end{array}$ & $\begin{array}{l}4 \cdot 10^{-5} \\
9 \cdot 10^{-5} \\
1.4 \cdot 10^{-4} \\
1.6 \cdot 10^{-4}\end{array}$ & $\begin{array}{l}0 \\
5 \cdot 10^{-5} \\
10^{-4} \\
2 \cdot 10^{-4}\end{array}$ & $\begin{array}{l}10^{-5} \\
4 \cdot 10^{-5} \\
6 \cdot 10^{-5} \\
2.4 \cdot 10^{-4}\end{array}$ & $\begin{array}{l}2 \cdot 10^{-5} \\
3 \cdot 10^{-5} \\
8 \cdot 10^{-5}\end{array}$ & $\begin{array}{l}2 \cdot 10^{-5} \\
7 \cdot 10^{-5} \\
8 \cdot 10^{-5}\end{array}$ & $\begin{array}{l}6 \cdot 10^{-5} \\
9 \cdot 10^{-5}\end{array}$ & $10^{-4}$ & $1.1 \cdot 10^{-4}$ & $1.2 \cdot 10^{-4}$ \\
\hline$N_{i, j}$ & $\sim 16500$ & $\sim 8250$ & $\sim 5500$ & $\sim 4100$ & $\sim 3300$ & $\sim 2750$ & $\sim 2350$ & $\sim 2050$ & $\sim 1820$ & $\sim 1650$ & $\sim 1500$ & $\sim 1380$ \\
\hline $\mathrm{k}_{\mathrm{ij}}$ & 1 & 3 & 4 & 6 & 8 & 9 & 11 & 13 & 14 & 16 & 18 & 19 \\
\hline
\end{tabular}

Relation (3) equal "1" (minor difference from condition (4)) for two satellite pair $\mathrm{A}_{2}-\mathrm{C}_{3}$ and $\mathrm{A}_{1}-\mathrm{C}_{4}$ (fig. 7) among 78 satellite pair combinations, which were created after satellites separation from dispenser.

Orbit planes of satellite $\mathrm{A}_{2}-\mathrm{C}_{3}, \mathrm{~A}_{1}-\mathrm{C}_{4}$ in some vicinity of orbit number $N \sim N_{i, j}$ have minor angular separation (to 20-30 ang. sec). The values of differences of relative separation velocities projections along flight direction $\Delta V_{V_{i, j}}$ and along the normal to $\mathrm{OX}_{1}$ axis of dispenser $\Delta V_{\perp V_{i, j}}$ for these satellite pairs are $\Delta V_{V_{i, j}}=\Delta V_{\perp V_{i, j}}=0.75 \mathrm{~m} / \mathrm{s}$. Distance between mentioned satellites pairs is about $\sim 0.5 \mathrm{~km}$ by the moment of their maximal approach in some vicinity of orbit number $N \sim N_{i, j}$ (fig. 6).
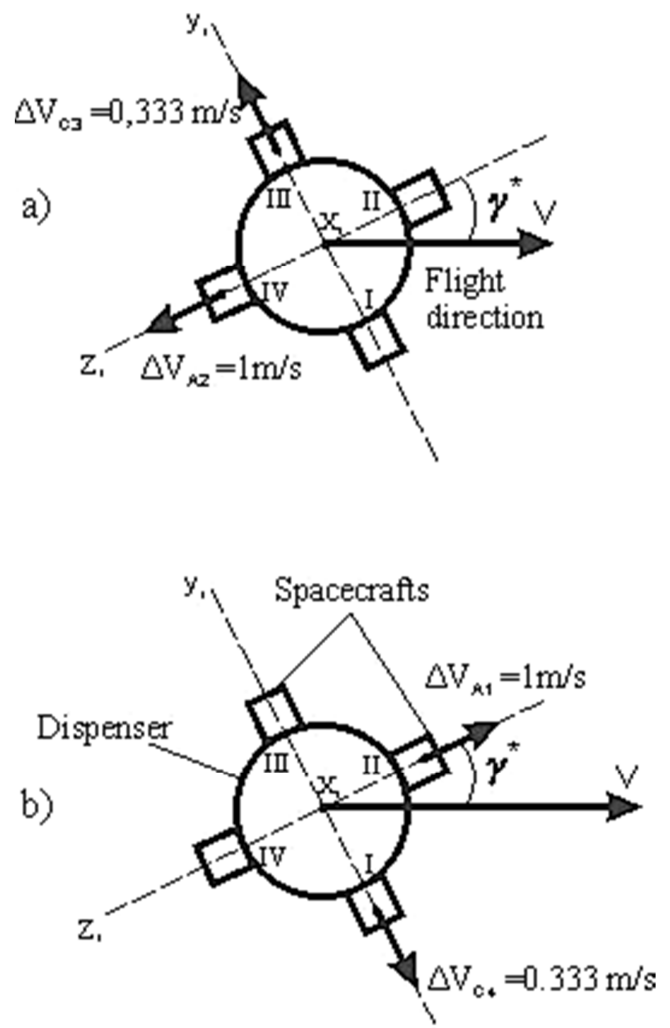

Fig. 7. Conditions of satellite pairs separation $\left(\Delta V_{V_{i, j}}=0.75 \frac{\mathrm{m}}{\mathrm{s}}, \Delta V_{\perp V_{i, j}}=\right.$ $0.75 \mathrm{~m} / \mathrm{s}$ )a) pairA $_{2}-C_{3}$. b) pairA $_{1}-C_{4}$.
Values of inaccuracies for satellite separation parameters $\delta\left(\Delta V_{V_{i, j}}\right)$ and $\delta\left(\Delta i_{i, j}\right)$ were determined due to carried out calculation results. These inaccuracies correspond to maximal satellites approaches to distances 9 and $12 \mathrm{~m}$ (fig. 8 points "• "). Thus $1 / 3$ of objects from all satellite number in constellation (fig. 2, 7) can approach to distances about $\sim 10 \mathrm{~m}$.

Area $\mathrm{D}\left(\left(\Delta V_{V_{i, j}}\right), \delta\left(\Delta i_{V_{i, j}}\right)\right)$ of inaccuracies is also showed at fig. 8. Its bounds correspond to satellites approach to $100 \mathrm{~m}$ (it was conditionally accepted that distance $100 \mathrm{~m}$ between satellites is acceptable boundary point of their autonomous flight including existing separation inaccuracies, physical nature of movement, calculation model imperfective and other factors).

Behavior of altitude inclinations $\Delta H$, geodetic latitudes $\Delta B$ and longitudes $\Delta \lambda$ at the phase of satellite approach to minimal distances are showed at fig. 9 a-c (the moment of satellite pair maximal approach is marked with 〈ڤ»)

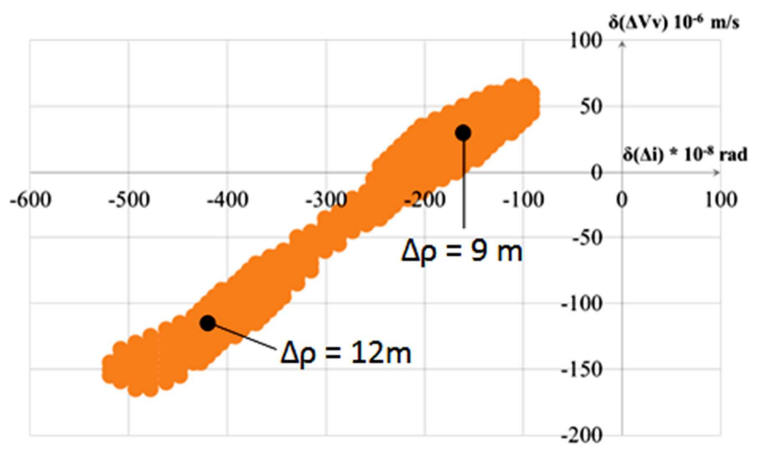

Fig. 8. Area of satellite approach critical values.

* Origin of coordinates corresponds to calculating parameters of satellite separation $\Delta V_{V_{i, j}}=0,75 \mathrm{~m} / \mathrm{s}$ and $\Delta i_{i, j}=10^{-4} \mathrm{rad}$.

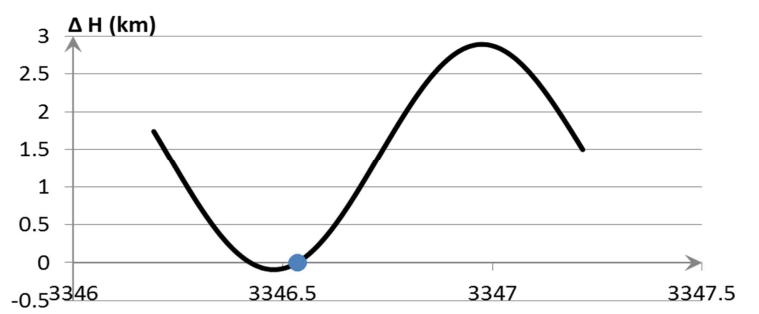

a) 


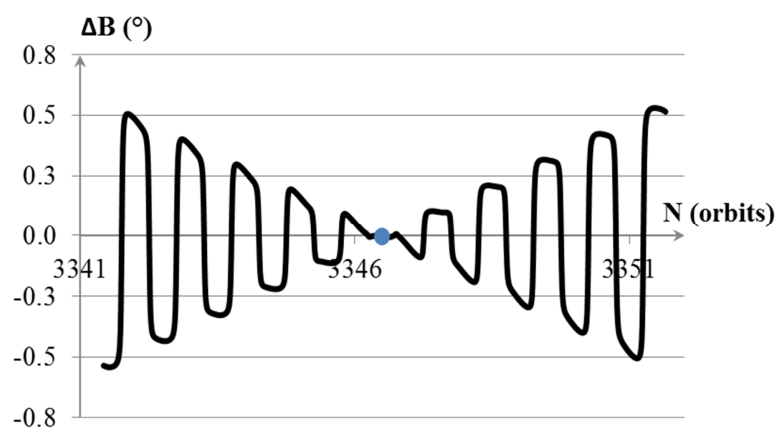

b)

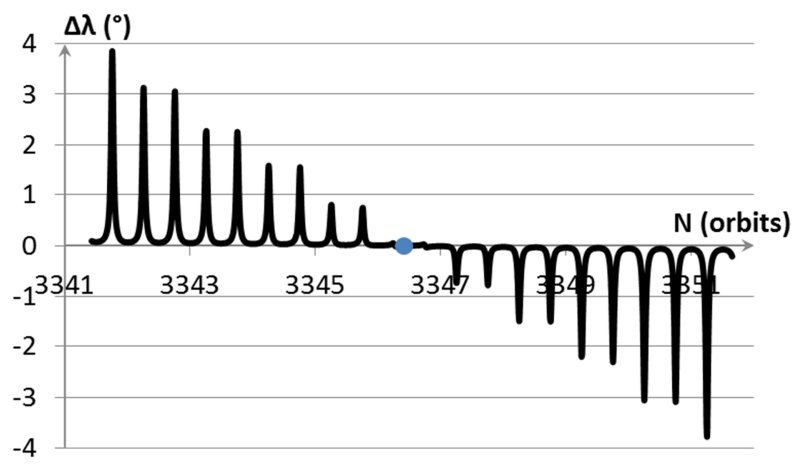

c)

Fig. 9. Range of orbital parameters change of satellites pair $\left.A_{2}-C_{3} a\right)$ by altitude $\Delta H b$ ) by latitude $\Delta B$ c) by longitude $\Delta \lambda$.

\section{The Alternative Separation Scheme for Big Constellation of Satellites}

The implementation of satellites separation scheme by fig. 2 is based on the usage of mechanical, electrical, pneumatic and other separation appliances. It is obvious, that such appliances have significant scattering of satellites separation operation (about 10\% from nominal) because of absence of power adjusting hardware. As a result there will be marked change of satellites separation conditions and deviation of further distances between them at the beginning of their autonomous flight and in further. In this case providing of satellites non-closing in constellation (or part of them) above mentioned bound of minimal distances between satellites in some vicinity of number $N \sim N_{i, j}$ sometimes can lead some difficulties.

Scheme at fig. 10 can be used as alternative one (satellites are separated serially normal to dispenser longitudinal axis in case of rocket moving with minor longitudinal acceleration along flight direction $\dot{w}_{x_{1}} \sim 0,02-0,1 \mathrm{~m} / \mathrm{s}^{2}$ ).

The advantage of this scheme is possibility of every further satellite separation by the control system instructions after achieving determined increase of flight velocity. Required characteristics of satellites constellation mutualrelative motion at the first phase after separation (including realization of tracing their flight trajectories on the safety distance $\left(y_{a c c}\right)$ from gas flows, which are formed by racing of thruster) and at further phases of their approaches are provided by following:

- Longitudinal rocket acceleration $\dot{w}_{x_{1}}$;

- Durations of satellites separation time intervals $\tau_{i, j}$;

- Satellites separation velocities normal to longitudinal rocket axis $\dot{y}_{0_{i}}$.

Imposition of velocity increment $\Delta V_{V_{i, j}}$ restriction for two $(i, j)$ satellites, which are separated serially, provides their non-closing in some vicinity of orbit number $N \sim N_{i, j}$ to distances that are lower than determined distance bounds. For satellites safety flight in the gas flows zone this restriction can be determined by relation (5) (fig. 10):

$$
\dot{y}_{0_{i}} \geq y_{a c c} \sqrt{\frac{\dot{w}_{x_{1}}}{2 x_{0_{i}}}}(5)
$$

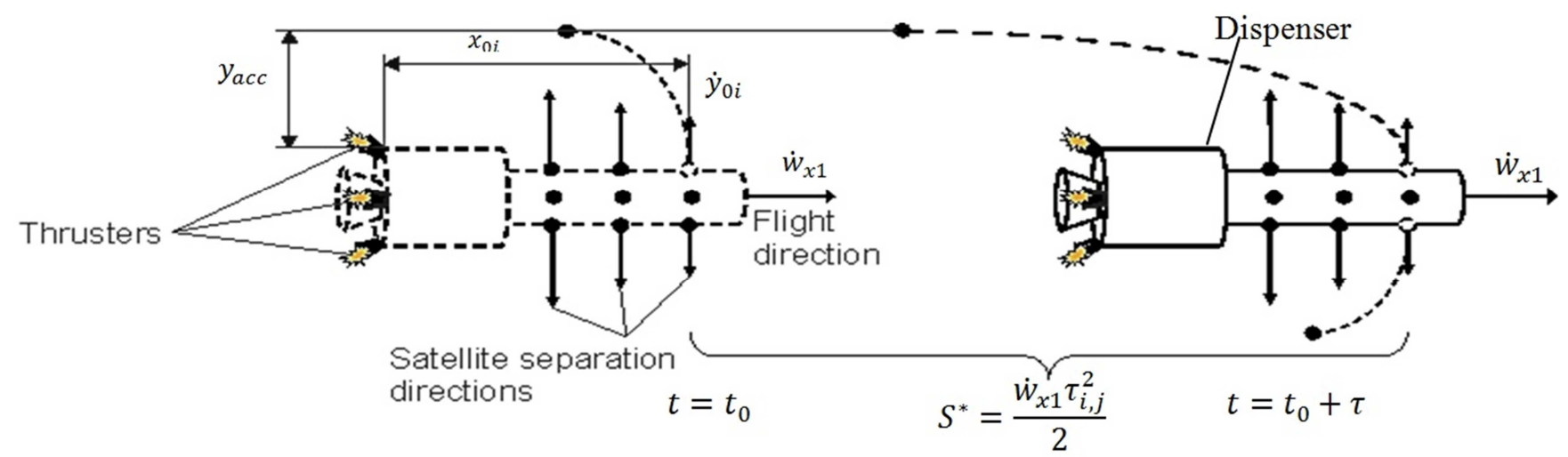

Fig. 10. Alternative separation scheme for big constellation of satellites

[2] Degtyarev A. Research of trajectories of spacecraft cluster injection into different orbits in one «Cyclone-4» vehicle launch//Degtyarev A., Degtyarenko P., Sirenko V., Sheptun A.//Space research in Ukraine 2010-2012.-Kyiv.

\section{References}

[1] Эльясберг, П. Е. Введение в теорию полета искусственных спутников Земли // Павел Ефимович Эльясберг // - М.: Наука, 1965. - 540с.
[3] Degtyarev A. Feasibility study of spacecraft cluster lunches with lunch vehicle into various basic orbits // Degtyarev A., Sheptun A., Mashtak I. // IAC-13-D2.P.16, 64th International Astronautical Congress, China, 2013. 
[4] Vorobiova I. Launch vehicle energy performance during single injection of the satellites into individual orbits // Vorobiova I., Sheptun A. // IAC-14-D2.2.9, 65th International Astronautical Congress, Canada, 2014.
[5] ВоробьеваИ. А. Проектно-баллистические исследования по групповым запускам спутников в одном пуске ракеты носителя на разнесенные базовые // Воробьева И. А., Шептун А.Д. // Научный журнал ХУПС, № 3, 2014. 